\title{
Politique
}

Politique

\section{Et si l'amour ne suffisait pas...de Nancy Guberman, Pierre Maheu et Chantal Maillé, Montréal, Les Éditions du Remue-ménage, 1991, 266 p.}

\section{Micheline Plasse}

Numéro 21, hiver 1992

URI : https://id.erudit.org/iderudit/040722ar

DOI : https://doi.org/10.7202/040722ar

Aller au sommaire du numéro

Éditeur(s)

Société québécoise de science politique

ISSN

0711-608X (imprimé)

1918-6584 (numérique)

Découvrir la revue

Citer ce compte rendu

Plasse, M. (1992). Compte rendu de [Et si l'amour ne suffisait pas...de Nancy

Guberman, Pierre Maheu et Chantal Maillé, Montréal, Les Éditions du

Remue-ménage, 1991, 266 p.] Politique, (21), 180-183.

https://doi.org/10.7202/040722ar d'utilisation que vous pouvez consulter en ligne.

https://apropos.erudit.org/fr/usagers/politique-dutilisation/ 
Et si l'amour ne suffisait pas...

de Nancy Guberman, Pierre Maheu et Chantal Maillé, Montréal, Les Éditions du Remue-ménage, 1991, 266 p.

Nul doute que cette recherche, fruit d'un travail collectif, a le grand mérite de mettre à nu les sacrifices, l'abnégation et l'inégalité du partage du fardeau humain et social que constitue le soin à donner à des personnes ágées ou souffrant de troubles mentaux sévères. Le lecteur ne sera pas étonné de constater qu'encore une fois ce sont le plus souvent les femmes qui paient le prix de la désinstitutionnalisation. Ainsi, les auteurs réussissent bien à nous montrer à quel point le quotidien de ceux qui s'occupent de personnes non autonomes peut être difficile et peut constituer un combat constant. Pour certains, malgré l'amour, la mort constitue la seule issue.

Plus précisément, les auteurs tentent de cerner les modalités, les dimensions de la prise en charge, le soutien sur lequel peuvent compter les personnes aux prises avec cette réalité et, enfin, les motifs qui peuvent amener des individus à s'engager dans des entreprises qui se révèlent être à long terme une tåche trop lourde pour un ou quelques-uns des proches du malade.

C'est à partir d'entrevues individuelles semi-dirigées de personnes vivant des situations de prise en charge de proches que les auteurs nous montrent les formes multiples que prend ce travail, ce qu'il implique comme tâches et ce qui est particulier a la prise en charge d'un adulte comparativement 
à celle d'un enfant. Ainsi, c'est à juste titre, croyons-nous, que les auteurs

s'inscrivent en faux contre une
conception de la prise en charge qui
en fait quelque chose de "naturel" et
d'allant de soi. Cette conception
tient davantage du mythe que de la
réalité. Comme toute autre activité
sociale, la prise en charge est
traversée par les rapports sociaux,
notamment les rapports de sexe
(p. 12).

Ainsi, les témoignages recueillis montrent d'une façon éloquente l'immense solitude et la somme d'énergie qui peut être déployée par des personnes prises dans des situations bien souvent dramatiques. A ce titre, les auteurs auraient pu davantage exploiter cette matière, et l'analyser plus en profondeur pour faire des recoupements et enrichir la synthèse. En effet, les témoignages parlent d'eux-mêmes, et le lecteur n'a pas trop de mal à se convaincre du pathétique des situations. En somme, une analyse plus poussée aurait pu éviter cette impression de redondance et mis davantage en relief les éléments que contenaient ces témoignages.

Le lecteur aurait aussi apprécié que ces témoignages soient confrontés avec ceux des «institutions» et ceux des responsables de cette politique de désinstitutionnalisation qui amène certainement des abus. Dans cette optique, le sujet aurait été davantage replacé dans son contexte. De même, dans le but d'élargir la discussion et la réflexion, il aurait été intéressant d'examiner comment d'autres sociétés, d'autres pays ont trouvé des réponses plus satisfaisantes à ce problème de soins à donner à de grands malades. D'autre part, il aurait aussi été intéressant et peut-être porteur d'un certain espoir de nous dire si, au Québec, des expériences alternatives ont pu être tentées avec succès. Pour l'étranger, les auteurs citent l'expérience hollandaise; plus de précisions sur cette expérience aurait sans doute contribué à imaginer qu'il y a peut-être une lumière au bout du tunnel. 
En revanche, les auteurs montrent qu'encore une fois l'insuffisance, voire l'absence de dialogue et de communications entre les divers types de ressources constitue un gaspillage de moyens que notre société endettée réussit quand même à se payer. Mais redisons-le, et les auteurs le montrent bien, le prix payé est aussi un prix caché, occulté et qui se mesure a l'aune des symptômes que sont l'épuisement, l'anxiété, la panique, la colère, l'angoisse, la frustration, la déprime, l'insomnie et la fatigue chronique.

En ce sens, cet ouvrage illustre pertinemment un partage inéquitable d'un fardeau trop lourd à porter pour une seule personne ou même par quelques-unes. A cet égard, le lecteur aurait apprécié avoir plus d'explications sur l'absence des hommes. Néanmoins, premier jalon d'une démarche plus poussée, l'ouvrage réussit à montrer l'ampleur du problème.

Par contre, dans un deuxième temps, il sera aussi essentiel de resituer ces vies faites de sacrifices dans le cadre des méandres de ces politiques gouvernementales qui en arrivent à créer ces situations aberrantes. La démarche entreprise devrait donc être poursuivie afin d'examiner des solutions possibles en tenant compte du rôle et des responsabilités de tous les acteurs, autant publics que privés.

II faudra aussi comparer notre expérience avec des expériences étrangères significatives. II $n$ 'en reste pas moins qu'il fallait faire ce premier pas et donner la parole aux sansvoix victimes des carences de nos politiques afin que des réalités trop longtemps tues interpellent ceux qui doivent l'être. Cet ouvrage constitue donc une référence essentielle à tous ceux qui ont à cœur l'amélioration de la qualité de vie des personnes qui s'en occupent. Bien écrit et facile d'accès, les auteurs ont donc réussi à "démontrer l'aspect "contraint et contraignant" du rôle joué par les femmes dans la prise en charge de leurs proches, voulant remettre en question le caractère supposément "naturel" de cette pratique» (p. 225).

Espérons que cet ouvrage sera suffisamment dérangeant pour que nos responsables politiques avec l'aide des administrateurs publics se penchent sérieusement sur cette problématique afin qu'il ne soit plus possible dans nos sociétés d'abuser des personnes qui prennent leurs responsabilités et qui du même coup cachent l'absence des 
solidarités nécessaires. Pour cette raison et bien d'autres, l'entreprise des auteurs doit être poursuivie et encouragée.

Micheline Plasse Université du Québec à Montréal 